\title{
LEGO-like assembly of peelable, deformable components for integrated devices
}

\author{
Sangkyu Lee ${ }^{1,5}$, Jaehwan $\mathrm{Ha}^{1,5}$, Sungjin $\mathrm{Jo}^{2}$, Junghyun Choi ${ }^{3}$, Taeseup Song ${ }^{1}$, Won Il Park ${ }^{1}$, John A Rogers ${ }^{4}$ \\ and Ungyu Paik ${ }^{3}$ \\ The development of various technologies has led to the advent of a variety of deformable devices. Despite significant \\ technological advancement in this area, it is still challenging to integrate different devices due to limitations such as substrate \\ issues and differences among growth and deposition conditions. Creating an interconnection between two different devices \\ currently requires the use of metallic wires/lines to build electrical connections. Here, we demonstrate a LEGO-like assembly of \\ the free-standing film of individually operable components encapsulated in a polymer overcoat layer, leading to the production \\ of an integrated architecture without additional electrical connections. The free-standing components are produced by the \\ peeling-off process. The sticky nature of the polymer layer enables the construction of supercapacitor arrays and simple RLC \\ circuits by interlocking the individual components. We expect that this approach will enable the fabrication of a variety of \\ custom-built devices using a LEGO-like assembly method.
}

NPG Asia Materials (2013) 5, e66; doi:10.1038/am.2013.51; published online 11 October 2013

Keywords: assembly; flexible; integration; peeling-off; supercapacitors

\section{INTRODUCTION}

Progress in various technologies that can release mechanical stress has enabled the production of various electronic circuits on non-conventional, deformable substrates including plastic, elastomeric rubber, fabric, paper and even human skin and living bodies. ${ }^{1-4}$ In general, these substrates can be easily deformed into a variety of shapes, even under small external forces. However, mechanical deformation leads to the formation of cracks in materials deposited on the substrate, deteriorating device performance and even leading to the fracture of the material or device layer. ${ }^{5}$ There are two primary approaches to endure and release stress under mechanical deformation. The first approach is to use soft materials in device fabrication, including organic semiconductors, ${ }^{6-8}$ carbon nanotubes ${ }^{9,10}$ and graphene. ${ }^{11,12}$ In addition, engineered polymers can be blended with such soft materials to achieve processability and further improve mechanical properties, as well as to compensate for some drawbacks and limitations of these technologies. For example, Someya and colleagues $^{7,8}$ fabricated stretchable organic transistors using elastic conductors by blending carbon nanotubes with elastomeric rubber, achieving a superior conductivity of $100 \mathrm{~S} \mathrm{~cm}^{-1}$ under $100 \%$ stretching. The other promising approach is to fabricate specially designed structures that are able to endure an applied strain based on the concept of out-of-plane motion in thin layers and to apply them to elastomeric substrates. ${ }^{13}$ Such specially designed structures are fabricated using general semiconductor processing techniques, followed by transfer printing on a desired substrate using an elastomeric stamp. This process of 'transfer printing' enables the fabrication of highly deformable structures and devices with rigid materials exhibiting high performance.

In this contribution, we demonstrate a novel assembly of freestanding, deformable electronic components into integrated electronic circuits. The assembly process is described in Figure 1. The device fabrication begins with the deposition of metal electrodes on an $\mathrm{SiO}_{2}$ wafer, followed by the formation of an active layer on the patterned electrodes. To produce a free-standing film, a polymer solution is applied over the entire substrate, as shown in Figure 1a for the case of carbon nanotube (CNT)-based supercapacitors. After the polymer overcoat is dried, the devices can be peeled off along with the polymer matrix, resulting in peelable and deformable devices (Figures $1 \mathrm{~b}$ and c). This peeling-off process relies on the adhesion force between the metal layer and the mother substrate as well as the elasticity of the polymer matrix, as in transfer printing. ${ }^{14}$ However, transfer printing mainly requires specialized wafers such as Si-on-insulator wafers or multistacked wafers. ${ }^{15}$ Unlike transfer printing, this approach enables

\footnotetext{
${ }^{1}$ Division of Materials Science Engineering, Hanyang University, Seoul, Republic of Korea; ${ }^{2}$ School of Energy Engineering, Kyungpook National University, Daegu, Republic of Korea; ${ }^{3}$ WCU Department of Energy Engineering, Hanyang University, Seoul, Republic of Korea and ${ }^{4}$ Department of Materials Science \& Engineering, University of Illinois at Urbana-Champaign, Urbana, USA

${ }^{5}$ These authors contributed equally to this work.

Correspondence: Professor JA Rogers, Department of Materials Science \& Engineering, University of Illinois at Urbana-Champaign, Urbana, USA

E-mail: jrogers@illinois.edu

or Professor U Paik, Department of Energy Engineering, Hanyang University, 17 Haengdang-dong, Seongdong-gu Seoul 133-791, Seoul 133-791, Republic of Korea. E-mail: upaik@hanyang.ac.kr

Received 25 June 2013; revised 10 August 2013; accepted 12 August 2013
} 
a
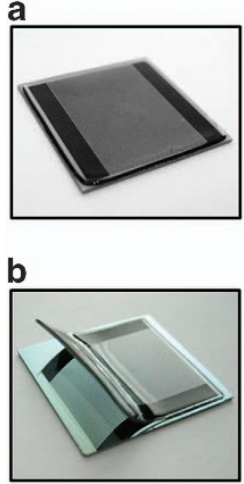

C

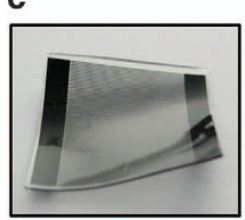

d

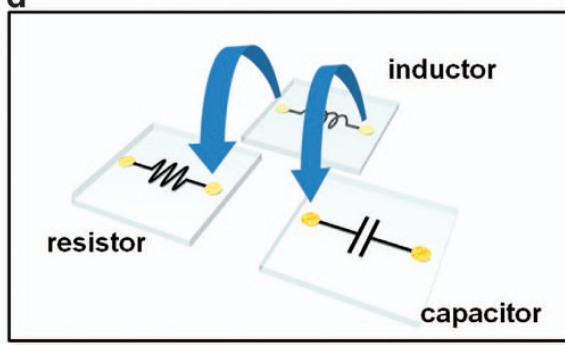

e

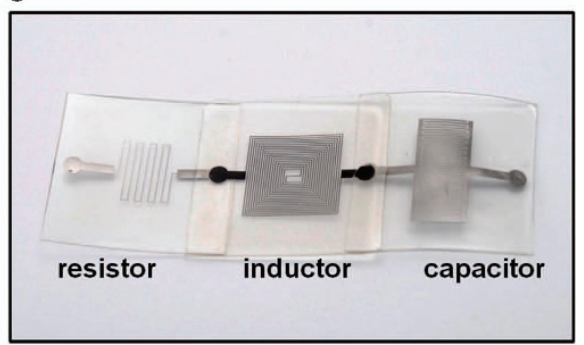

Figure 1 Processing steps for the fabrication of peelable devices and LEGOlike assembly of electronic circuits. (a) Electrochemical capacitors fabricated on a wafer encapsulated in a polymer electrolyte layer. (b) The process of peeling off the capacitors. (c) Flexible, peelable electrochemical capacitors. (d) LEGO-like assembly. The arrows indicate the direction of integration. (e) A simple RLC circuit using these methodologies.

device fabrication using the very inexpensive and common $\mathrm{SiO}_{2}$ wafer substrate. Additionally, this method requires neither an additional etching process to release the structures nor subsequent processes of inking, retrieving and transfer printing with a polydimethylsiloxane (PDMS) stamp. The polymer encapsulating the entire layout of the device during the peel-off process acts as a substrate and thus eliminates the need for an additional substrate. Moreover, the functionality of the polymer can be tuned by blending other functional materials exhibiting mechanical, electronic, optical and thermal properties, which could lead to a significant improvement in device performance or enable the development of a new class of functional devices.

In previous approaches, complex, multifunctional devices and their arrays are constructed in-plane according to a specific design determined in advance before the fabrication. In some cases, the fabricated objects or devices are fixed on the substrate with a layer of organic adhesives (epoxy resins or photocurable polymers). ${ }^{16}$ In contrast, in the current approach, the stickiness of the polymer matrix is used to assemble various free-standing devices via simple stacking and subsequent pressing with fingers, allowing for the bottom-up assembly of these components into integrated architectures. This approach of utilizing the intermolecular stickiness of a polymer seems to be similar to the previous technique of laminating two elastomeric layers containing split pieces of a device. ${ }^{17,18}$ For example, in the case of an organic thinfilm transistor, source and drain electrodes were formed on an elastomeric layer, and then this layer was laminated against another substrate containing the remaining parts of the gate electrode, dielectric layer and organic semiconductor. These two layers adhered strongly to each other via the dehydration reaction between the silanol groups belonging to the two layers. This assembly concept can extend the concept of lamination to enable the integration of different devices that can be operable individually

even before the lamination process, creating a more straightforward approach to the bottom-up assembly. Figures $1 \mathrm{~d}$ and e describe the bottom-up device construction. The basic passive electronic components of the inductor, resistor and capacitor were fabricated as freestanding components via the fabrication steps explained above. The devices were arranged based on the intended application, with Figure 1d demonstrating how to assemble the different devices into an integrated circuit: a simple RLC series circuit. By following the arrows in panel $\mathrm{d}$, the circuit shown in panel e can be easily fabricated. This demonstration holds out promise that users will be able to design and make circuits for their own purposes by assembling the individual circuit components.

\section{EXPERIMENTAL PROCEDURES}

\section{Mechanical properties}

Three different metal layers of molybdenum $(\mathrm{Mo})$, copper $(\mathrm{Cu})$ and gold $(\mathrm{Au})$ were formed on an $\mathrm{SiO}_{2} / \mathrm{Si}$ wafer (thickness of $\mathrm{SiO}_{2}: 200 \mathrm{~nm}$ ) using $\mathrm{RF}$ sputtering $(\mathrm{Mo})$ or e-beam evaporation $(\mathrm{Cu}$ and $\mathrm{Au}$ ). The adhesion force between each metal layer and the underlying $\mathrm{SiO}_{2}$ layer on the $\mathrm{Si}$ wafer was evaluated with a scratch tester (J\&L Tech, Ansan-si, Korea). In this study, a poly(vinyl alcohol) (PVA) layer was utilized. The aqueous PVA solution was prepared by dissolving PVA $(\mathrm{Mw}=89000-98000,99+\%$ hydrolyzed, Sigma Aldrich, St Louis, MO, USA) in deionized water at $90^{\circ} \mathrm{C}$ for $3 \mathrm{~h}$. Then, the solution was poured into a petri dish and dried overnight in an oven at $60^{\circ} \mathrm{C}$. The dried PVA film was easily detached after drying. To control the mechanical properties of the PVA layer, different amounts of phosphoric acid were added to the aqueous PVA solution. PVA films containing phosphoric acid $(99.999 \%$, Sigma Aldrich) were prepared using the same procedure. The mechanical properties of those PVA films were tested using an Autograph AGS-J500N universal testing machine (UTM) (Shimadzu, Kyoto, Japan). The adhesion of the PVA film to the metal layer was also measured using the UTM. For this experiment, PVA solutions containing different concentrations of phosphoric acid were poured on the $\mathrm{SiO}_{2}$ wafers deposited with different metal layers (Mo, $\mathrm{Cu}$ and $\mathrm{Au}$ ).

\section{Peeling-off process}

Growth of CNTs. The regions for electrode pads were defined on an $\mathrm{SiO}_{2} / \mathrm{Si}$ wafer via photolithography. A molybdenum (Mo) layer (thickness: $300 \mathrm{~nm}$ ) and a bilayer catalyst $(\mathrm{Al} / \mathrm{Fe}=10-20 / 1-5 \mathrm{~nm})$ were sequentially deposited by RF sputtering and e-beam evaporation, respectively, followed by a lift-off process in an acetone bath. Vertically aligned multiwalled carbon nanotubes (MWNTs) were grown on the regions of patterned catalyst using a thermal chemical vapor deposition process. Before the growth of the MWNTs, the catalyst was annealed at the growth temperature of $650-800^{\circ} \mathrm{C}$ under an $\mathrm{Ar}$ flow of $300 \mathrm{sccm}$. MWNTs were grown by flowing acetylene source gas (flow rate: $5-25 \mathrm{sccm}$ ) and a mixed carrier gas of hydrogen and argon (total flow rate: $\left.300-600 \mathrm{sccm}, \mathrm{H}_{2} / \mathrm{Ar}=1: 1 \mathrm{v} / \mathrm{v}\right)$. The morphology of the CNTs was observed using a JSM-7600F scanning electron microscope (JEOL, Tokyo, Japan).

Preparation of gel electrolyte. An aqueous PVA solution was prepared by dissolving $1 \mathrm{~g}$ of PVA in $10 \mathrm{ml}$ of deionized water at $90^{\circ} \mathrm{C}$ for $3 \mathrm{~h}$. Phosphoric acid $(0.8 \mathrm{~g})$ was mixed with the aqueous PVA solution, yielding the gel electrolyte solution.

Fabrication of free-standing supercapacitors. Sufficient gel electrolyte solution was applied over the patterns of CNT grown on the wafer to cover the patterns. The gel electrolyte was dried overnight in an oven at $60^{\circ} \mathrm{C}$. After drying, the gel electrolyte film was peeled off the wafer, yielding free-standing supercapacitors. The infiltration of the gel electrolyte into the voids between CNTs was confirmed by scanning electron microscope.

Measurement of electrochemical performance. The precise masses of MWNTs were estimated using a Sartorius S2 microbalance (resolution $0.1 \mu \mathrm{g}$, Sartorius, Göttingen, Germany). Cyclic voltammetry (CV) curves were obtained in the 
voltage rage of $0-0.8 \mathrm{~V}$ at a scan rate of $100 \mathrm{mV} \mathrm{s}^{-1}$ using a Parstat 2273 potentiostat/galvanostat (Princeton Applied Research, Oak Ridge, TN, USA). Galvanostatic charge/discharge cycles were carried out at various current rates (1-5 $\mathrm{Ag}^{-1}$ ) using a 660B electrochemical analyzer (CH Instruments, Austin, TX, USA). The specific capacitance of the supercapacitor $\left(C_{\mathrm{sp}}\right)$ was calculated from the results of galvanostatic charge-discharge tests using the following equation: ${ }^{19}$

$$
C_{\mathrm{sp}}=\frac{i \Delta t}{m \Delta E}
$$

where $i$ is the discharge or charge current, $\Delta t$ is the discharge time, $m$ is the mass of part of an MWNT electrode and $\Delta E$ is the potential window $(0-0.8 \mathrm{~V})$. Cycle performance was measured for up to 1000 cycles. After 1000 cycles, the supercapacitors were peeled off the wafer, and then the cycle performance of the peeled supercapacitors was measured over 1000 cycles. The current rate was $1.0 \mathrm{Ag}^{-1}$

Mechanical deformation. Mechanical deformation includes bending, folding and stretching. The influence of bending and folding on the electrochemical performance of supercapacitors was evaluated by CV. The CV measurements were carried out under bending and folding deformations. A repeated bending test was also performed using a customized automated bending stage. The bending curvature was $R=1 \mathrm{~cm}$. The galvanostatic charge-discharge tests were performed at a specific bending cycle and were used to calculate the specific capacitances after bending. The stretching test was performed using a custommade jig. The galvanostatic charge-discharge measurements were carried out with increasing applied strains up to $50 \%$. The stretch and release test was repeated five times.

\section{LEGO-like assembly}

Supercapacitor array. Five peelable supercapacitors were fabricated separately and then assembled via the LEGO-like assembly method, yielding a supercapacitor array in which five supercapacitors are connected in series. A filmtype solar cell (PowerFilm, Ames, IA, USA) was used to charge the supercapacitor array. Solar cells were charged under controlled illumination for $100 \mathrm{~s}$. After the illumination was turned off, the voltage of the supercapacitor was measured using a B1500A semiconductor device analyzer (Agilent, Santa Clara, CA, USA). The charged supercapacitor array was also used to turn-on a commercial green light-emitting diode (LED). The current-voltage characteristics of the LED were measured using the semiconductor device analyzer (Supplementary Figure S1).

RLC simple circuits. Passive components, including a resistor, an inductor and a capacitor, were fabricated on the $\mathrm{SiO}_{2} / \mathrm{Si}$ wafer. Each device layout is presented in Supplementary Figure S2. The patterns of the Mo and bicatalyst layers were formed via a photolithography, deposition and lift-off process. After the growth of the CNTs and the application of the gel electrolyte layer, the whole film containing the passive device was peeled off the wafer. The characteristics of each passive component were tested using a WaveJet 354A oscilloscope (LeCroy, Chestnut Ridge, NY, USA) by applying a sinusoidal voltage generated by a 33120A function generator (Agilent), as shown in Supplementary Figure S3. High-pass and low-pass filters were fabricated with a peeled resistor and capacitor. Their transient responses to a square input were measured using the oscilloscope.

Measurement of contact resistance. Two polymer films containing Mo electrodes were prepared through a peeling-off process and then assembled. The resistance of the metal electrodes was measured using an Agilent 34401A multimeter, as shown in Supplementary Figure S4.

\section{RESULTS AND DISCUSSION}

The mechanism of the peeling-off process can be explained in the following three ways. First, during the solidification of the solvent-cast polymer films, the films undergo drying-induced stress due to volume changes, ${ }^{20}$ which could affect the underlying layer. Second, there is competition between the adhesion strengths at the two interfaces, between the polymer overcoat and the metal layer and between the metal layer and the substrate. The final factor is the mechanical properties of the dried polymer film. The adhesion strength between the metal layer and the underlying substrate was measured using a scratch test. Three different metal layers, including molybdenum $(\mathrm{Mo})$, copper $(\mathrm{Cu})$ and gold $(\mathrm{Au})$, were formed on an $\mathrm{SiO}_{2}$ wafer using RF sputtering (Mo) and an e-beam evaporation ( $\mathrm{Cu}$ and $\mathrm{Au})$. The results of the adhesion tests are summarized in Supplementary Table S1. The mechanical properties of the dried polymer films were also measured using a UTM. PVA was used as a polymer layer because it is a water-soluble, non-toxic synthetic polymer with an excellent film-forming ability and excellent mechanical properties. ${ }^{21}$ In this study, the mechanical properties of PVA were controlled by using phosphoric acid as a plasticizer agent. The addition of phosphoric acid to PVA leads to complexation with PVA molecules, decreasing the crystallinity of PVA. ${ }^{22}$ These changes increase the flexibility of the resulting PVA films. Supplementary Figure S5 shows the strain-stress curves for the resulting PVA films. The pure PVA film exhibits a high tensile strength and Young's modulus, but it only exhibits elastic behavior at low levels of strain. Increasing the concentration of phosphoric acid in PVA films elongates the elastic regions and leads to decreases in the tensile strength and Young's modulus. These results indicate that PVA films with different mechanical properties can be prepared by controlling the dosage of phosphoric acid. To correlate these factors, PVA solutions containing different concentrations of phosphoric acid were poured onto the $\mathrm{SiO}_{2}$ wafers deposited with different metal layers $(\mathrm{Mo}, \mathrm{Cu}$, and $\mathrm{Au}$ ). After drying, a peeling test was performed. These results are summarized in Figure 2. The $x$ and $y$ axes indicate the measured Young's moduli of the PVA films and the adhesion strengths of metals on the $\mathrm{SiO}_{2}$ wafers, respectively. The colors indicate the force required to peel the PVA film with its underlying metal layer from the $\mathrm{SiO}_{2}$ wafer. As anticipated, complete peeling was achieved easily when the polymer overcoat had a high Young's modulus and the adhesion strength between the metal layer and the $\mathrm{SiO}_{2}$ wafer was low. Previously, the gold layer was detached due to the weak adhesion of the gold layer to the wafer and the application of a chemical adhesive (thiol-terminated molecule) between the gold layer and the PDMS stamp. ${ }^{23}$ Note that metal layers with high adhesion can be peeled off by controlling the mechanical properties of the polymer layer. The dotted circles in Figure 2 indicate the conditions under which the PVA overcoats with underlying metal layers were freely detached after drying. This

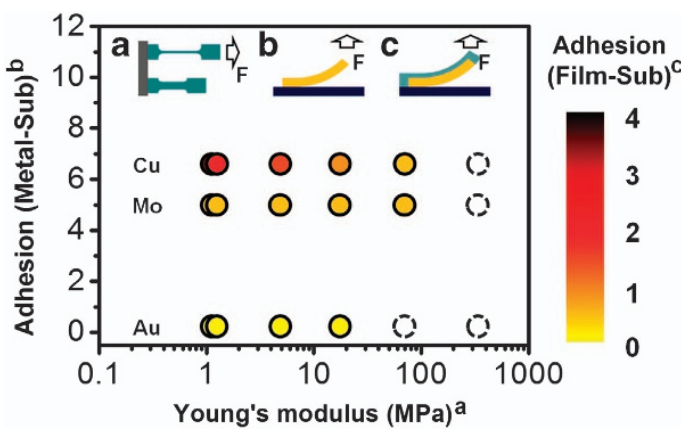

Figure 2 The mechanism for the fabrication of peelable components. The $x$ and $y$ axes show the Young's modulus of PVA films and the adhesion strength between the metal layer and the $\mathrm{SiO}_{2}$ wafer, respectively. The color index on the right side indicates the adhesion force between the metal layer encapsulated by PVA film and the $\mathrm{SiO}_{2}$ wafer. The inset presents schematic illustrations describing the measurements of each value. The unit of adhesion is the Newton ( $\mathrm{N})$. 
phenomenon is only observed when the Young's modulus of the dried PVA film is high. This free detachment is attributed to the contribution of drying-induced stress in the PVA film. This stress is related to the physical properties of polymer films including the Young's modulus ${ }^{20}$ and the glass transition temperature. ${ }^{24}$ Croll $^{20}$ reported the relationship between the Young's modulus of a polymer and the stress developed during the drying process. The drying stress increases with an increasing Young's modulus of the polymer and a decreasing volume fraction of retained solvent in the film. Dried, pure PVA film exhibits a high elastic modulus (Supplementary Figure S5) and has little water content. Considering the relationship proposed by Croll, ${ }^{20}$ a pure PVA film is expected to generate the highest drying stress compared with other PVA films containing phosphoric acid. Phosphoric acid decreases the glass transition temperature of the polymer due to the plasticization effect, ${ }^{22}$ leading to a decrease in the drying-induced stress. ${ }^{24}$ The drying stress also increases significantly when most of the water in the polymer film, generally $80-90 \%$, is evaporated. ${ }^{24}$ Therefore, the presence of a large amount of water in the PVA film with phosphoric acid also prevents the generation of stress.

Using this peeling-off technique, we demonstrate the fabrication of a peelable, deformable energy storage device. We select CNT-based supercapacitors due to their importance as energy storage devices together with lithium ion batteries. The fabrication of the freestanding supercapacitor is illustrated in Supplementary Figure S6. First, regions for electrode pads were formed on the $\mathrm{SiO}_{2}$ wafer via general photolithography. A molybdenum (Mo) layer $(\sim 300 \mathrm{~nm}$ thick) was sputtered. The electrode pads were formed after a lift-off process in an acetone bath. These pads connect supercapacitors with a galvanostat/potentiostat apparatus for the evaluation of device performance or with other devices such as energy-harvesting devices and light-emitting diodes. They also serve as current collectors similar to the typical two-electrode configurations for stacked supercapacitors. In this work, we fabricated the planar supercapacitors such that their two electrodes faced each other in-plane. Especially for planar supercapacitors, nanomaterials with a large specific surface area should be utilized as electrode materials to ensure efficient use of the limited substrate area. ${ }^{25,26}$ We constructed electrodes with the comb-like geometry that is commonly used in planar supercapacitors. ${ }^{25,26} \mathrm{Next}$, the $\mathrm{Al} / \mathrm{Fe}$ bilayer catalyst was formed on the wafer using an e-beam evaporator. Then, vertically aligned MWNTs were selectively grown on the catalyst pattern through a chemical vapor deposition process with acetylene carbon source gas and mixed carrier gases ( $\mathrm{Ar}$ and $\mathrm{H}_{2}$ ) at $650-800^{\circ} \mathrm{C}$. The onedimensional structure of $\mathrm{CNT}$ arrays can significantly increase the total surface area of the electrode, providing ions with accessible surfaces. After growth, an aqueous gel electrolyte solution containing phosphoric acid and PVA was poured over the wafer with grown CNTs. Subsequently, the gel electrolyte was dried in an oven at $60^{\circ} \mathrm{C}$. The gel electrolyte fully infiltrated into the voids between carbon nanotubes (Supplementary Figure S7) to encapsulate the entire planar supercapacitors composed of CNT electrodes and electrode pads. This complete wetting of the electrode materials with the gel electrolyte increased the effective surface area to which ions are accessible, producing supercapacitors with a high specific capacitance. ${ }^{27}$ After drying, the edge of the gel electrolyte layer was grabbed with standard tweezers and then the layer was mechanically peeled off from the wafer. The entire planar supercapacitors were also peeled off along with the gel electrolyte layer. Note that the peeling-off process demonstrated in this study can be used to fabricate a free-standing, independently operable device. a
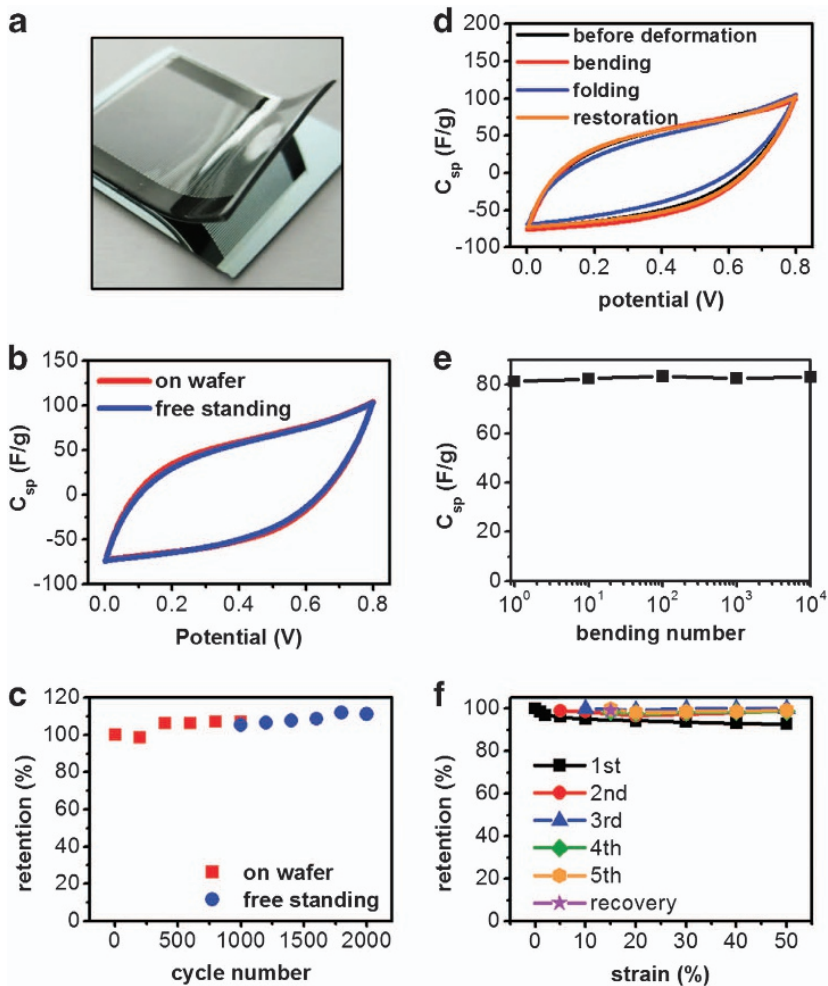

Figure 3 Demonstration of the peeling-off process for the fabrication of electrochemical capacitors. (a) A photograph of electrochemical capacitors. (b) Cyclic voltammetry (CV) curves of capacitors on the wafer and after detachment from the wafer, that is, as free-standing films. (c) Cycle performance for capacitors on the wafer and subsequent cycle performance after detachment. (d) CV curves for the supercapacitors following each mechanical deformation. (e) Specific capacitance as a function of bending repetitions (up to 10000). (f) Specific capacitances measured at different strains; the values were also measured after releasing the applied strain. This test was repeated five times.

Figure 3 presents the electrochemical properties of the resulting peelable supercapacitors. We compared the electrochemical performance of supercapacitors fabricated on an $\mathrm{SiO}_{2}$ wafer (hereafter, we refer to this device as a planar supercapacitor) with that of peeled, free-standing supercapacitors. First, planar supercapacitors were fabricated by pouring the gel electrolyte on the patterned MWNT electrodes, followed by drying. The electrochemical performance of these devices was characterized by $\mathrm{CV}$, galvanostatic charge/discharge and cycle performance. Figure 3 a presents a picture of supercapacitors in the process of being peeled off the wafer. After evaluation, the supercapacitors were peeled the substrate to obtain the peeled, freestanding supercapacitors. The CV curves were obtained in the voltage range from 0 to $0.8 \mathrm{~V}$ at a scan rate of $100 \mathrm{mV} \mathrm{s}^{-1}$. Figure $3 \mathrm{~b}$ compares the CV curves of the planar supercapacitors with those of the resulting peeled supercapacitors. The peelable supercapacitors exhibit a CV curve highly similar to that of the planar supercapacitors. These results confirm that the peeling-off process does not affect the supercapacitive behavior of our devices. This fact can also be observed from the galvanostatic charge-discharge curves of the two supercapacitors. Supplementary Figure S8a presents the galvanostatic charge-discharge behavior of these devices at an increasing current rate from 1 to $5 \mathrm{Ag}^{-1}$. For the planar supercapacitors, the voltage drop at initial discharge was $13.6 \mathrm{mV}$ at a current density of $1 \mathrm{Ag}^{-1}$ 
(Supplementary Figure S8b), indicating very low equivalent series resistance; for the peelable supercapacitors, the drop was $17.2 \mathrm{mV}$ (Supplementary Figure S8c). The specific capacitances of these supercapacitors $\left(C_{\mathrm{sp}}\right)$ were calculated from the results of the galvanostatic charge-discharge tests at different current densities; these results are presented in Supplementary Figure S9. At a current density of $1 \mathrm{Ag}^{-1}$, planar supercapacitors exhibited a specific capacitance of $89 \mathrm{Fg}^{-1}$, whereas the peelable supercapacitors yielded a specific capacitance of $101 \mathrm{Fg}^{-1}$. This increase can be related to the activation of the electrode with repeated charge-discharge cycles. ${ }^{28}$ In addition, we evaluated the cycle performance, which is a main characteristic of supercapacitors, at a current density of $1 \mathrm{Ag}^{-1}$. The cycle test for planar supercapacitors was performed by conducting 1000 charge and discharge cycles. After 1000 cycles, the planar supercapacitors were peeled off the wafer to fabricate peelable supercapacitors. The test was resumed for another 1000 cycles. The specific capacitances were plotted as a function of cycle number, as shown in Figure $3 \mathrm{c}$. The results indicate that both the planar and peelable supercapacitors have excellent cycle characteristics.

The effect of mechanical deformation on the electrochemical performance of peelable, free-standing supercapacitors was also investigated. Mechanical deformation includes bending, folding and stretching. The flexible nature of the gel electrolyte film enables the supercapacitors to be bent, folded and stretched, as shown in Supplementary Figure S10. The demonstration used glass tubes $(R=3.3$ and $8.7 \mathrm{~mm})$ and a glass slide (thickness $=1.1 \mathrm{~mm})$. In addition, the stretchability of the supercapacitors was tested using a jig modified from a clamp. Figure $3 \mathrm{~d}$ presents the CV curves for peelable supercapacitors under bending and folding deformations. All measurements were performed in the deformed state. Under bending, the CV curve remained unchanged. Folding caused a slight change in the CV curves, but releasing the mechanical deformation restored the $\mathrm{CV}$ curve to its original state. These results show that such deformations hardly affect the device performance. To observe changes in device characteristics resulting from repeated bending, the galvanostatic charge-discharge test was carried out after the bending test. The bending test was performed using a customized automated bending stage. The bending curvature was $R=1 \mathrm{~cm}$. After bending, the specific capacitances were calculated from the results of the galvanostatic charge-discharge test. As shown in Figure $3 \mathrm{e}$, the peelable, free-standing supercapacitors exhibited a nearly constant specific capacitance, even after 10000 cycles of the bending test. The galvanostatic charging and discharging behaviors were measured under different applied tensile strains, and the corresponding specific capacitances were calculated. The results are plotted in Figure $3 \mathrm{f}$. Stretching with a strain below $10 \%$ dropped the capacitance to $\sim 90 \%$. Further elongation slowly decreased the specific capacitance. At $50 \%$ strain, $\sim 93 \%$ of the initial specific capacitance was retained. Strain relaxation restored $\sim 99 \%$ of the initial specific capacitance and $\sim 95 \%$ of the original dimensions. The reversibility of this stretchability was also investigated by repetitive stretching and release. After four cycles of stretching and release, both the specific capacitance and dimensions of the supercapacitors were stabilized. These behaviors are attributed to the restructuring of the nanostructure in the film with a

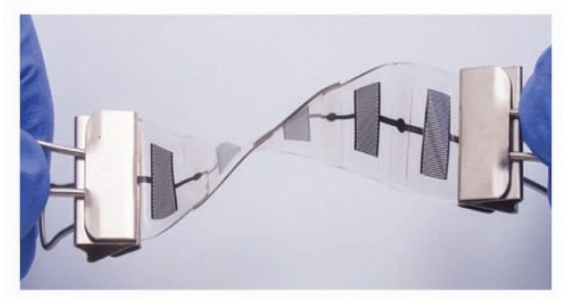

C
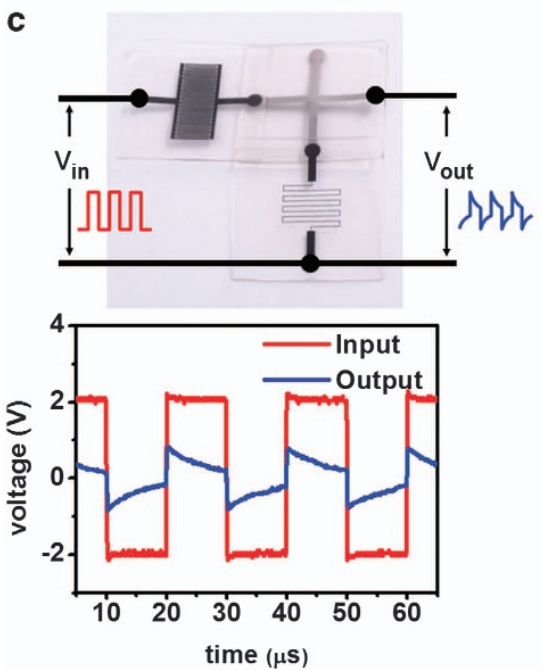

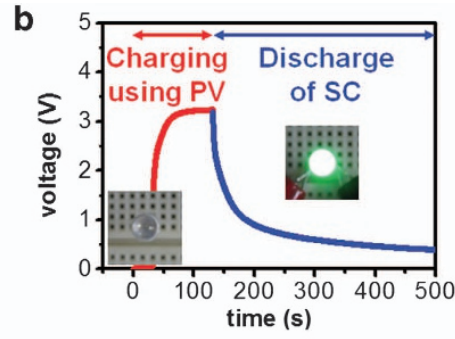

d
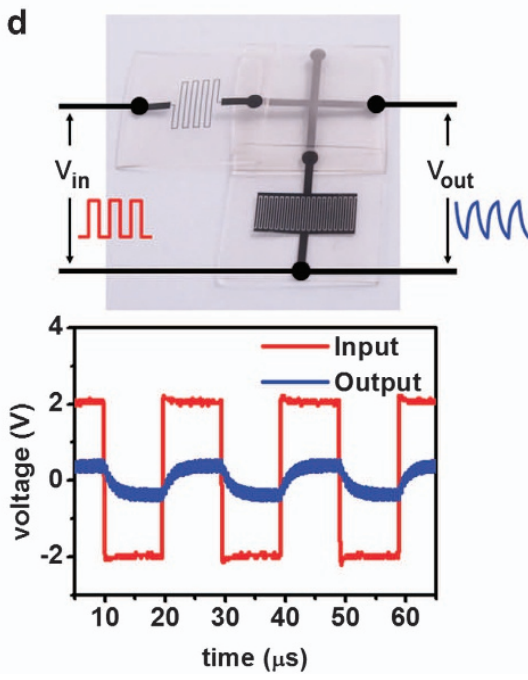

Figure 4 LEGO-like assembly of peelable components. (a) A photograph of twisted supercapacitor arrays assembled with five free-standing supercapacitors in series. (b) Time-voltage curves describing the charging of supercapacitors using a solar cell and consecutive discharging of charged supercapacitors. The inset photograph shows a commercial green LED powered by the energy stored in the supercapacitors. (c, d) RC circuits for a high-pass filter (panel c) and a low-pass filter (panel d) fabricated using a peelable resistor, a capacitor and a connector. The upper images show the circuit structures, and the lower graphs present the transient responses of the filters to a square input. 
stretching. ${ }^{17}$ After the first deformation, the inner structures can be stabilized so that the properties of the supercapacitors remain unchanged. The presence of phosphoric acid in the PVA film caused the formation of gel-like PVA networks, which yielded a slightly elastic film and consequently provided limited stretchability to the peelable, free-standing supercapacitors. The application of this stress-releasing structure ${ }^{29}$ and the modification of the physicochemical properties of the polymer layer could increase the stretchability.

PVA is inherently sticky, ${ }^{30}$ and its stickiness increases in the presence of water molecules, as water is a ubiquitous plasticizer that lowers the glass transition temperature of the polymer, thus increasing the stickiness. ${ }^{31}$ Phosphoric acid also functions as a plasticizer for $\mathrm{PVA}^{22}$ and leads PVA to hold large amounts of water inside the network, increasing the stickiness of PVA films containing phosphoric acid. This stickiness can have the same role as that of the silanol groups that combine two layers of elastomers in the lamination process. ${ }^{17,18}$ This study shows that this stickiness can be used to assemble individual peeled, free-standing electronic components into an integrated circuit. Five free-standing supercapacitors were fabricated using the peeling-off process. These supercapacitors were assembled using the sticky nature of the film. Figure 4a shows the array of supercapacitors that were connected in series. This array is robust and can endure mechanical deformation such as twisting. Additionally, this supercapacitor array can be charged with solar cells. Figure $4 \mathrm{~b}$ shows the charging and discharging voltage profiles of the supercapacitors. Solar cells generate electrical current, which simultaneously charges the supercapacitors. The supercapacitors are fully charged after charging for $\sim 100 \mathrm{~s}$, producing an output voltage of $\sim 3.2 \mathrm{~V}$. Fully charged, integrated supercapacitors can turn-on a commercial LED, as shown in the inset of Figure $4 \mathrm{~b}$. This demonstration utilized a commercial green LED (a turn-on voltage of $\sim 2.8 \mathrm{~V}$ was obtained from I-V curve shown in Supplementary Figure S1). An LED consumes less electricity than other electrical loads. Using the LEGO-like assembly method developed here, the total capacitance and output voltage of the energy storage device (supercapacitors) can be modulated by varying the number of supercapacitors and the design of the series and parallel circuits, enabling these integrated energy storage devices to meet the requirements of various electrical loads including LED arrays or other devices.

This concept can also be used to fabricate electronic circuits from individual electronic components. Individual passive electronic components such as resistors, inductors and capacitors were fabricated on the $\mathrm{SiO}_{2}$ wafer. The peeling-off process was used to prepare the corresponding free-standing components. These components can be assembled to make a simple RLC circuit. When a sinusoidal voltage was applied to a passive electronic component, as schematically shown in Supplementary Figure S3a, the resistor yielded a decreased current (Supplementary Figure S3b), whereas the inductor and capacitor led to phase shifts in the output current, as shown in Supplementary Figures S3c and S3d, respectively. These results indicate that the peeled RLC components work well under an alternating current field. High- and low-pass filters can be constructed using these components. The first-order high- and low-pass filters consist of the peeled resistor and capacitor, which are shown in Figures $4 \mathrm{c}$ and $\mathrm{d}$. A circuit in which an input voltage is applied to the capacitor first and in which the output voltage is taken across the resistor (which is connected in series with the capacitor) acts as a high-pass filter (differentiator). The reverse arrangement produces a low-pass filter (integrator). In these experiments, an alternating current voltage with $2 \mathrm{~V}$ of amplitude was applied, and its frequency was $50 \mathrm{kHz}$. The high-pass filter transformed the square wave input signal to a spike-shaped waveform (Figure 4c, lower graph), whereas the low-pass filter generated a wave-shaped waveform from the same input signal (Figure 4d, lower graph).

\section{CONCLUSION}

In conclusion, the peeling-off process and LEGO-like assembly demonstrated here provide an alternative methodology to produce deformable devices. Unlike conventional approaches, this methodology does not require specially designed expensive wafers, an etching process or adhesives to adhere devices or architectures to the substrate. Additionally, the polymer layer used in this demonstration can be formulated with functional materials, which could improve the device performance and create new classes of devices. The technique developed here enables the fabrication of peelable, free-standing supercapacitors that are mechanically deformable, and it also allows for the integration of these individual free-standing components into an integrated circuit, providing the opportunity to fabricate customized devices using pre-fabricated components. We expect that more complex, multifunctional circuits or architectures could be fabricated by applying the peeling-off process to various components, including diodes, transistors and their integrated circuits.

\section{CONFLICT OF INTEREST}

The authors declare no conflict of interest.

\section{ACKNOWLEDGEMENTS}

This work was supported by the Global Research Laboratory (GRL) Program (K20704000003TA050000310) through the National Research Foundation of Korea (KRF) funded by the Ministry of Science, ICT (Information and Communication Technologies) and Future Planning, and the International Cooperation program of the Korea Institute of Energy Technology Evaluation and Planning (KETEP) grant funded by the Korea government of Ministry of Trade, Industry \& Energy (2011T100100369).

1 Kim, D. H., Ahn, J. H., Choi, W. M., Kim, H. S., Kim, T. H., Song, J. Z., Huang, Y. G. Y., Liu, Z. J., Lu, C. \& Rogers, J. A. Stretchable and foldable silicon integrated circuits. Science 320, 507-511 (2008)

2 Kim, D. H., Kim, Y. S., Wu, J., Liu, Z. J., Song, J. Z., Kim, H. S., Huang, Y. G. Y., Hwang, K. C. \& Rogers, J. A. Ultrathin silicon circuits with strain-isolation layers and mesh layouts for high-performance electronics on fabric, vinyl, leather, and paper. Adv. Mater. 21, 3703-3707 (2009).

3 Kim, D. H., Viventi, J., Amsden, J J., Xiao, J L, Vigeland, L., Kim, Y S., Blanco, J. A , Panilaitis, B., Frechette, E. S., Contreras, D., Kaplan, D. L., Omenetto, F. G., Huang, Y. G., Hwang, K. C., Zakin, M. R., Litt, B. \& Rogers, J. A. Dissolvable films of silk fibroin for ultrathin conformal bio-integrated electronics. Nat. Mater. 9, 511-517 (2010).

4 Kim, D. H., Lu, N. S., Ma, R., Kim, Y. S., Kim, R. H., Wang, S. D., Wu, J., Won, S. M., Tao, H., Islam, A., Yu, K. J., Kim, T. I., Chowdhury, R., Ying, M., Xu, L. Z., Li, M., Chung, H. J., Keum, H., McCormick, M., Liu, P., Zhang, Y. W., Omenetto, F. G., Huang, Y. G. Coleman, T. \& Rogers, J. A. Epidermal electronics. Science 333, 838-843 (2011).

5 Wu, H., Hu, L. B., Carney, T., Ruan, Z. C., Kong, D. S., Yu, Z. F., Yao, Y., Cha, J. J., Zhu, J., Fan, S. H. \& Cui, Y. Low reflectivity and high flexibility of tin-doped indium oxide nanofiber transparent electrodes. J. Am. Chem. Soc. 133, 27-29 (2011)

6 Gelinck, G. H., Huitema, H. E. A., Van Veenendaal, E., Cantatore, E., Schrijnemakers, L., Van der Putten, J. B. P. H., Geuns, T. C. T., Beenhakkers, M., Giesbers, J. B., Huisman, B. H., Meijer, E. J., Benito, E. M., Touwslager, F. J., Marsman, A. W., Van Rens, B. J. E. \& De Leeuw, D. M. Flexible active-matrix displays and shift registers based on solution-processed organic transistors. Nat. Mater. 3, 106-110 (2004).

7 Sekitani, T., Noguchi, Y., Hata, K., Fukushima, T., Aida, T. \& Someya, T. A rubberlike stretchable active matrix using elastic conductors. Science 321, 1468-1472 (2008).

8 Sekitani, T., Nakajima, H. Maeda, H., Fukushima, T., Aida, T., Hata, K. \& Someya, T. Stretchable active-matrix organic light-emitting diode display using printable elastic conductors. Nat. Mater. 8, 494-499 (2009).

9 Cao, Q., Kim, H. S., Pimparkar, N., Kulkarni, J. P., Wang, C. J., Shim, M., Roy, K. Alam, M. A. \& Rogers, J. A. Medium-scale carbon nanotube thin-film integrated circuits on flexible plastic substrates. Nature 454, 495-500 (2008). 
10 Sun, D. M., Timmermans, M. Y., Tian, Y., Nasibulin, A. G., Kauppinen, E. I., Kishimoto, S., Mizutani, T. \& Ohno, Y. Flexible high-performance carbon nanotube integrated circuits. Nat. Nanotechnol. 6, 156-161 (2011).

11 Kim, R. H., Bae, M. H., Kim, D. G., Cheng, H. Y., Kim, B. H., Kim, D. H., Li, M., Wu, J., Du, F., Kim, H. S., Kim, S., Estrada, D., Hong, S. W., Huang, Y. G., Pop, E. \& Rogers, J. A. Stretchable, transparent graphene interconnects for arrays of microscale inorganic light emitting diodes on rubber substrates. Nano Lett. 11, 3881-3886 (2011).

12 Lee, S. K., Kim, B. J., Jang, H., Yoon, S. C., Lee, C., Hong, B. H., Rogers, J. A., Cho, J. H. \& Ahn, J. H. Stretchable graphene transistors with printed dielectrics and gate electrodes. Nano Lett. 11, 4642-4646 (2011).

$13 \mathrm{Kim}$, D. H., Xiao, J. L., Song, J. Z., Huang, Y. G. \& Rogers, J. A. Stretchable, curvilinear electronics based on inorganic materials. Adv. Mater. 22, 2108-2124 (2010).

14 Meitl, M. A., Zhu, Z. T., Kumar, V., Lee, K. J., Feng, X., Huang, Y. Y., Adesida, I., Nuzzo, R. G. \& Rogers, J. A. Transfer printing by kinetic control of adhesion to an elastomeric stamp. Nat. Mater. 5, 33-38 (2006).

15 Yoon, J., Jo, S., Chun, I. S., Jung, I., Kim, H. S., Meitl, M., Menard, E., Li, X. L., Coleman, J. J., Paik, U. \& Rogers, J. A. GaAs photovoltaics and optoelectronics using releasable multilayer epitaxial assemblies. Nature 465, 329-333 (2010).

16 Sun, Y. G. \& Rogers, J. A. Inorganic semiconductors for flexible electronics. Adv. Mater 19, 1897-1916 (2007).

17 Loo, Y.-L., Someya, T., Baldwin, K. W., Bao, Z., Ho, P., Dodabalapur, A., Katz, H. E. \& Rogers, J. A. Soft, conformable electrical contacts for organic semiconductors: High-resolution plastic circuits by lamination. Proc. Natl. Acad. Sci. USA 99, 10252-10256 (2002).

18 Kim, J. B., Lee, S., Toney, M. F., Chen, Z., Facchetti, A., Kim, Y. S. \& Loo, Y.-L. Reversible soft-contact lamination and delamination for non-invasive fabrication and characterization of bulk-heterojunction and bilayer organic solar cells. Chem. Mater. 22, 4931-4938 (2010).

19 Wang, H., Zhang, L., Tan, X., Holt, C. M. B., Zahiri, B., Olsen, B. C. \& Mitlin, D. Supercapacitive properties of hydrothermally synthesized $\mathrm{CO}_{3} \mathrm{O}_{4}$ nanostructures. J. Phys. Chem. C 115, 17599-17605 (2011).

20 Croll, S. G. Origin of residual internal-stress in solvent-cast thermoplastic coatings. J. Appl. Polym. Sci. 23, 847-858 (1979).

21 Masuda, M. in Polyvinyl Alcohol - Developments (ed. Finch, C. A.) Ch. 12, 404-431 (Wiley, New York, USA, 1992).
22 Prajapati, G. K., Roshan, R. \& Gupta, P. N. Effect of plasticizer on ionic transport and dielectric properties of $\mathrm{PVA}-\mathrm{H}_{3} \mathrm{PO}_{4}$ proton conducting polymeric electrolytes. J. Phys. Chem. Solids 71, 1717-1723 (2010).

23 Smythe, E. J., Dickey, M. D., Whitesides, G. M. \& Capasso, F. A technique to transfer metallic nanoscale patterns to small and non-planar surfaces. ACS Nano 3, 59-65 (2009).

24 Wedin, P., Lewis, J. A. \& Bergström, L. Soluble organic additive effects on stress development during drying of calcium carbonate suspensions. J. Colloid Interface Sci. 290, 134-144 (2005).

25 Pech, D., Brunet, M., Durou, H., Huang, P. H., Mochalin, V., Gogotsi, Y., Taberna, P. L. \& Simon, P. Ultrahigh-power micrometre-sized supercapacitors based on onion-like carbon. Nat. Nanotechnol. 5, 651-654 (2010).

26 Gao, W., Singh, N., Song, L., Liu, Z., Reddy, A. L. M., Ci, L. J., Vajtai, R., Zhang, Q., Wei, B. Q. \& Ajayan, P. M. Direct laser writing of micro-supercapacitors on hydrated graphite oxide films. Nat. Nanotechnol. 6, 496-500 (2011).

27 Kaempgen, M., Chan, C. K., Ma, J., Cui, Y. \& Gruner, G. Printable thin film supercapacitors using single-walled carbon nanotubes. Nano Lett. 9, 1872-1876 (2009).

28 Lu, X. H., Zheng, D. Z., Zhai, T., Liu, Z. Q., Huang, Y. Y., Xie, S. L. \& Tong, Y. X. Facile synthesis of large-area manganese oxide nanorod arrays as a high-performance electrochemical supercapacitor. Energ. Environ. Sci. 4, 2915-2921 (2011).

29 Xu, S., Zhang, Y., Cho, J., Lee, J., Huang, X., Jia, L., Fan, J. A., Su, Y., Su, J., Zhang, H., Cheng, H., Lu, B., Yu, C., Chuang, C., Kim, T.-i., Song, T., Shigeta, K., Kang, S., Dagdeviren, C., Petrov, I., Braun, P. V., Huang, Y., Paik, U. \& Rogers, J. A. Stretchable batteries with self-similar serpentine interconnects and integrated wireless recharging systems. Nat. Commun. 4, 1543 (2013).

30 Liu, Q., Zhao, F., Dong, X. H., Yang, C. H. \& Chen, F. L. Synthesis and application of porous $\mathrm{Sm}_{0.2} \mathrm{Ce}_{0.8} \mathrm{O}_{1.9}$ nanocrystal aggregates. J. Phys. Chem. C 113, 17262-17267 (2009).

31 Adhikari, B., Howes, T., Bhandari, B. R. \& Truong, V. Stickiness in foods: a review of mechanisms and test methods. Int. J. Food Prop. 4, 1-33 (2001).

(c) (i) (2)(2) This work is licensed under a Creative Commons Attribution-NonCommercial-ShareAlike 3.0 Unported License. To view a copy of this license, visit http://creativecommons. org/licenses/by-nc-sa/3.0/

Supplementary Information accompanies the paper on the NPG Asia Materials website (http://www.nature.com/am) 